\title{
Neurophenomenology: A Perspective of Scientific Epistemology
}

\author{
Zhang Jingzhu \\ School of Marxism, Shenyang Jianzhu University \\ Shenyang, China, 110168 \\ 1058954011@qq.com
}

\author{
Ren Qiaohua \\ School of Marxism, Shenyang Jianzhu University \\ Shenyang, China, 110168 \\ tony805@163.com
}

\begin{abstract}
Since the 21st century, philosophical epistemology and methodology have been greatly affected by phenomenology. The most representative view is that in neuroscience research, there is a phenomenological method to illustrate consciousness. Firstly, neurophenomenology combines first-person phenomenological methods with third-person neuroscience methods to deal with "hard" problem. Secondly, from the perspective of scientific epistemology, phenomenology combines the standpoints of Foundherentist to explore the influence of the integration of neuroscience and phenomenology on science epistemology. It is found that neurophenomenology of consciousness reflects the implications of constructivist and methodologically embodies the necessity of combining scientific rationality with introspection training in scientific research.
\end{abstract}

Keywords-Neurophenomenology; Foundherentist; Scientific Epistemology; Methodology

\section{INTRODUCTION}

In the case of the consciousness analysis of phenomenology, the intuitive method starts from the category of consciousness and makes up for the limitations of the methods from natural science. In the late 20th century, phenomenology had an important influence on cognitive science, resulting in an embodied cognitive approach. Hubert L. Dreyfus argues that the ontological hypothesis by computational theory, representation theory and artificial intelligence is untenable so that it has the needs for integration of neuroscience with embodied-ontology [1]. This consciousness problem has spawned the birth of neurophenomenology. Neurophenomenology as a scientific research program aims to solve the problem of consciousness, in which the neuroscience and phenomenology organically are combined to study the experience, mind and consciousness, emphasizing the embodiment of human mind. Neurophenomenology is also regarded as a phenomenological study of psychology, closely related to neurocognics, neuroanology and behavioral neuroscience, so the question is raised with respect to how to interpret neurocognology and its contribution to epistemology?

\section{THE RISE OF NEUROPHENOMENOLOGY}

Neuro-phenomenology refers to a research program that addresses the problem of consciousness from the perspective of pragmatics, linking neuroscience to phenomenology, and is designed to study experience, mind and consciousness, and to emphasize the specific conditions of the human mind. This field is related to neuropsychology, neuroanology, behavioral neuroscience (also known as biological psychology), and psychopathological studies. In 1990, Laughlin C., McManus J. and Aqua E. proposed the theory of neurocognition. In the mid-1990s, the cognitive neuroticist, Francisco Varela gave the neuro-phenomenology to different connotations, which inspired many philosophers and neuroscientists to explore the problem in new directions.

Phenomenology is a philosophical method of daily experience. Phenomenology is derived from the Greek "phainomenon", meaning "displayed in the experience object", which is to verify the phenomenon pointing to different consciousness. This is so-callled the "first person" perspective of the consciousness. Thus, phenomenology is a discipline that examines how the phenomenon manifests for us and how we derive meaning from it [2]. From a historical point of view, Husserl is regarded as a well-known phenomenological philosopher with a specific methodology to study consciousness, that is, the suspension. Husserl is Brentano's student, who argues that from a view of mind, the recognition of consciousness is characterized by the intention, which is extremely important. The concept of intention is often interpreted as "directness"; consciousness is always directed at an object. In the mid-20th century, the philosopher Morris Mello Ponty developed an embodied-phenomenology. Neuroscience is seen as a brain science that explores the thirdperson approach to consciousness. Scientists engaged in conscious research argue that neither the first nor the third person method can solve the problem of consciousness.

Therefore, neuroscience and phenomenology began to seek the path between the intermediation, but after all, phenomenology and philosophy of mind have the ontological inconsistency, which causes a barrier for the dialogue between the two. Philosopher, Hubert Dreyfus, opens up a new direction in the integration of neuroscience with embodiedontological research through the attempt to solve the related problems through computationalism, representationism, 
cognitive science, and ontological assumptions of artificial sciences. However, we still have arguments against Hubert Dreyfus's explanation of Husserl, with a high interest in understanding how Husserl's phenomenology is integrated with psychology [3]. Typical representatives of neurocognological studies include Laughlin C. , McManus J., Varela F., etc., who try to analyze phenomenology with neuroscience. That is, the insight into phenomenology will be useful in interpreting the cognitive basis of biology.

\section{UNDERSTANDINGS OF NEUROPHENOMENOLOGY}

\section{A. The Defination of consciousness}

The consciousness can be classified into different categrories: creature consciousness, background consciousness and state consciousness, transitive or intransitive consciousness, access consciousness, phenomenal consciousness, introspective consciousness, pre-reflective selfconsciousness, etc. [4] However, we still have a hot debate on the problem of access consciousness and phenomenal consciousness. A view is that, from the perspective of phenomenology, the content of consciousness can not get access to thinking, which can not access to the rational control of action and oral report. Another view is that the concept of consciousness is incoherent. [5] From the perspective of phenomenology, some Asian phenomenological studies point to the concept of intentionality and pre-reflective selfconsciousness.

Pre-reflective self-consciousness is the original form of self-consciousness, belonging to any conscious experience: in addition to the object of intent, any experience instinctively characterize itself. This self-representation is a primitive form of self-consciousness that needs to be understood from three aspects: 1) it does not require any reflection or introspective follow-up with the object consciousness; 2) it does not form a belief or make a judgment; 3) From the spontaneous and voluntary point of view it is negative. In this regard, it is necessary to distinguish between the purely rational process of experience and the relationship between the content of the experience. Experience involves no more than the object's noema, and the tacit consciousness is treated as a process of intent [6]. For example, when we see an object, we also perceive someone's vision from a non-diverted, pre-reflective, and digestive perspective; when we visualize a mind image, we also realize that it is visualized. Tacit self-consciousness is often interpreted as a kind of non-objective physical consciousness, which is a reflection of the body or experiencerelated subjectivity. In addition to the object of consciousness, from the perspective of neurocognition, any credible theory of consciousness must explain this kind of subjective prereflective experience.

\section{B. The Definition of neurophenomenology}

Neuro-phenomenlogy argues that neuroscience needs to explain that the mental model we are experiencing is the image of the object and how the brain leads to a sense of selfidentity in the cognitive process. We feel the thought image that is formed in special circumstances [7]. From the phenomenological point of view, the second problem concerns with the purely rational side of consciousness, especially the minimal or core self brought by the subtle subjective experience, hereby making contrast with narrative or autobiographical self [8]. Cognitive scientists emphasize that this primitive self-consciousness is related to the regulation of life, the emotional process of the body, so all cognitive and intentional behavior is based on emotion, but this view has set off the debate on a sense of access consciousness and phenomenal consciousness. From the phenomenological point of view, life experience is composed of pre-verbal, prereflective and emotional state of mind, which can not directly get access to thinking, introspection and oral report. These states must be in a case of primitive self-consciousness, or they can not be characterized as consciousness; especially through the first person method, the mental state can get access to thinking, reflection, introspection and oral report.

In short, many theorists distinguish between access consciousness and phenomenal consciousness. Neurophenomenology, by emphasizing the structural dynamics of object-oriented objects, reconstructs the theoretical framework, including the experience of reflection or the structure and time dynamics of introspection.

\section{NEUROPHENOMENOLOGY : A VIEW OF SCIENTIFIC EPISTEMOLOGY}

\section{A. The Relationship Between Consciousness And Epistemological Research}

The phenomenological approach to cognitive cognition requires reference to linguistics and interdisciplinary theory, covering the scope of the phenomenon of consciousness, including the category under the label of consciousness and the etymology of linguistics. From the point of view of linguistics, consciousness is rooted in the Latin "conscius". From Indo-European, consciousness is called as Skei, which means that cognition needs to distinguish between cognitive subjects and objects [9]. The linguistic roots and definitions of consciousness revolve around a series of references, from the biological instincts to the experience, and the acquired environment, social and cultural abilities. Consciousness refers to the understanding and response of the organism to the environment, from the awakening of environmental awareness and self-consciousness, to the behavior and interactive ability, to the social and cultural thinking, information and characterization process, which can be said that consciousness on the basis of experience is subject to deepening. Consciousness revolves around many abilities, and these abilities are derived not only from physical, personal, social, and spiritual dimensions, but also from human perception, intentionality and organizational competence, as well as knowledge and unique symbolic characterization. In addition, consciousness comes from the ability to work with the 
external environment and to process, analyze and evaluate the information. Consciousness includes information that corresponds to thought and action, produces intentionality and completes goal-oriented behavior. Human consciousness also includes public levels-consciousness, practice, standards and assumptions about the world. Of course, consciousness also involves knowledge and information processing capabilities that guide the organism to act in an adaptive and meaningful way.

Baruss explains the different levels of consciousness and points to the three main forms of consciousness, namely, goaloriented behavior, subjective self-consciousness and intentionality [10]. This suggests that consciousness refers to a broader interaction and relationship between organisms' external behavior and internal cognitive environment. Consciousness is in the interactive relationship between the cognitive subject and the cognitive object, and is involved in the biological and cultural field in the interactive relationship between the realistic mind and the physical entities. Accordingly, the study of consciousness requires the integration of biological physiology and cultural semiotics to explore the relationship between consciousness epistemology on the subjective experience and the special conditions of physiological function, rather than reducing consciousness into brain structure or activity.

Of course, the importance of conscious research lies in the further development of epistemology. In general, epistemology focuses primarily on the nature of consciousness, and the commonality of consciousness and epistemology is derived from the ultimate concern of the world's cognitive characteristics. Cognitive nature, environment and social interaction, conceptual and representative ability, social relations and traditions, and goal-oriented behavior are the core of the essence of consciousness and epistemology. The state of change of consciousness is particularly important for the development of epistemology, since the state of change of consciousness provides different experiences from traditional epistemology and extends the phenomenological experience. Meditation traditions include activities, exercises and practices designed to construct experience with visual information, thus transcending common experiences and understanding the real construction process of reality.

\section{B. Neurophenomenology and Foundherentist}

In epistemology, foundherentist is a theory of confirmation that combines the two opposing theoretical elements that emphasize the infinite regression: foundherentist tends to be arbitrary, and coherence tends to be cyclical (Mincheshausen's triple dilemma problem). Susan Haack develops and maintains a basic theory of coherence in Evidence and Inquiry: Towards Reconstruction in Epistemology. Her thoughts stem from Bertrand Russell's epistemology, and coherence are elements of confirmation [11]. Fundamentalism is referred as basic beliefs can support derived beliefs unilaterally, that is, from the former to the latter. The idea of foundherentist is that faith can support each other when beliefs belong to the same coherent set of beliefs. However, with the refinement of these theories, some fundamentalists began to admit that the basic beliefs were wrong, and the derived beliefs were able to support each other. At the same time, some coherence theorists began to acknowledge that they should attach importance to empirical beliefs to reflect the coherence of reality or the degree of confirmation. Therefore, this interdependent theory begins to converge slowly: if the fundamentalists try to explore why the basic beliefs and derivative beliefs can not support each other, it is easy to move towards the coherence theoretical side; if the coherence theorists try to explore why attention is paid to the experience of faith, it is easy to fall to fundamentalism [12].

The philosopher Hacck's foundherentis epistemology is of great importance to Neurophenomenology. She combines the foundationalist and coherentist of traditional epistemology, and links the role of experience in empirical evidence with the support of different beliefs [11]. Foundherentist examines the empirical basis of beliefs, including the integration of beliefs. The basis of experience supports and confirms faith to a certain extent. Foundherentist does not guarantee truth, but stands in a critical position to measure the interpretation of truth. The standard derives from our theory of the world and its derivation from human cognition and its limitations. This kind of natural science knowledge is physiological and phenomenological, and in essence, it is of great significance to the study of neuro-phenomenology. In particular, Hacck's meta-epistemological naturalism is based on the standpoint of neuro-phenomenology to investigate the knowledge and phenomenal experience of human cognitive ability from the perspective of cognitive natural science. Experience and introspection become the ultimate basis for determining empirical beliefs, but these are not reliable. The phenomenological study of neuro-phenomenology or constructionist of biology is precisely the basis of the idea of foundherentist, with the depth probe into the theory of consciousness and meta-physics theory.

\section{Across-subject And Object Dichotomy - From a View of Constructivist}

The neuro-phenomenology was originally proposed by Laughlin, McManus and d'Aquili. It explores the reciprocal relationship between the biological substrate and phenomenological experience produced during the construction of the consciousness model and addresses the interdependence of consciousness in biology and experience domain, and attempts to circumvent the problem of mind perception as a unique, body-independent dualism [13]. Subsequently, Varela F. on the basis of the development of embodied enactive cognition made the neuro-phenomenology methods integrated into this framework, emphasizing the cognitive is embodied. The cognitive process depends on the physical characteristics of the body, and the body resides in the environment, providing a cognitive content, while the nervous system and the whole organism are autonomical [14].

The neuro-phenomenology approach examines the nature of consciousness and knowledge along two dimensions: one is to point out the correspondence between the role of the brain and the nature of the consciousness from the neuroticism, and the other is the correspondence of the spiritual phenomenon, the cognitive hypothesis and its construction and phenomenological experience. These two dimensions 
contribute to the formation of the basis of consciousness, and the activities of the cognitive subject are closely related to the level of symbolic cognition and the level of biophysical behavior. It can be said that the study of neurophenomenology is an attempt to avoid the perception as a unique, independent of the body. It develops a phenomenological domain framework for human experience to provide a biological basis. Neuro-phenomenology regards the mind and the brain as the intrinsic structural representation of the human body. It can be said that human knowledge and experience constitute a comparatively symbolic form of character, but it does not deny a reality of the external environment, but points to cognition that human beings can use symbolic forms and genres to understand the external and internal reality. The phenomenological approach to neurophenomenology or biology provides a methodology of understanding the nature of consciousness and a meta-physics perspective. The physical and spiritual world we understand and perceive include the mind and the body of the operational environment of cognitive model. Our pattern of consciousness and our world model are based on human mind activities, which need to distinguish between the operational world and the cognitive model from the perspective of analytics, that is, to distinguish between the external objective world and the perceptible and empirical phenomenal world.

The neuro-phenomenology approaches are used based upon the following requirements: First, it investigates the commonality of cross-species cognitive model from the perspective of brain information processing capacity. Second, it takes the core role of the activity (practice), especially the intervention of various different states of consciousness as the core of the construction of the consciousness model. Similar thoughts have been explained in Piaget's epistemology. Piaget's distinction between anatomy and physiology (structure and function) is considered to be an important basis for epistemology, which summarizes the following dimensions: 1) psychoanatomy or structure; 2) determining the commonness and diversity of the mind Psychoanalysis; 3) psychophysiology, the study of spiritual function [15]. Accordingly, the study of human cognitive neurophenomenology method requires the use of structural, behavioral and empirical information and other means. This structure includes neurological and semantic systems, especially in neurophysiology; observable behaviors are regulated by organism nerves and regulation of biological structures; the experience is formed in observation and direct participation in events, hereby generating knowledge from it. Therefore, the biological structure of the organism, cognitive structure and behavior are the source of consciousness, while knowledge is derived from the interaction between the subject and object.

Laughlin, McManus and d'Aquili recognize the role of the genetic epistemology in explaining the formation of cognition. In their view, epistemology of neuro-phenomenology must be reflected in an evolutionary sense, including three cognitive processes, namely, ontogenetic process, phylogenic process and social interactive process. These three processes represent: 1) brain formation processes; 2) a constant pattern of neuropsychological development; and 3) application of social conditions in the context of the environment. These three factors always affect the human observation at the same time, and they are interactive in the construction of the cognitive environment.[16] This approaches to neuro-phenomenology are combined brain neurobiology with cognitive structure (individual, social, cultural constructs) from the perspective of consciousness and experience. The relationship between the cognitive subject and the cognitive object constitutes the interaction between the brain, experience and behavior, so that the organism has the consciousness. This empirical study points directly to two empirical approaches: introspection and neuroscience approaches. In other words, the rise and essence of consciousness can be understood by virtue of integrating the inner experience of the cognitive subject with consciousness of the brain (consciousness organ). The approach of neuro-phenomenology points to two functions: one is the interaction function of neural cognition and learning, and the other is the function of coordinating the interaction of human biology in physical, social and cultural environment. The epistemological foundations of the phenomenological approach need to integrate the theory of neurology and social culture and require a hypothetical metaphysical metaphor of materialism to balance the phenomenological epistemology of idealism by recognizing the structural essence of experience and cognition.

\section{CONCLUSION}

Firstly, special relationships between human cognitive experience and cultural interaction are built up. Human organizational level corresponds with operational environment and cognized environment. The operational level points to Kant's ontology, which is independent of human knowledge or perception and is a transcendent reality.[17] In other words, the limitations of the human nervous system is that for all real ontologies and operable worlds, unknown areas are far more than known areas. For mankind, the operable world is a cognitive environment or world. Human perceived cognitive worlds and externally manipulative world experiences are the result of neural activity patterns that arise from physiological parameters and acquisition systems. The congenital supernatural structure of the organism is produced by means of the environment and the cultural experience, such as the shamanism, the verge of death, the interpretation of the dream, and the psychodynamics, all of which tend to be a special cognitive pattern. The human response to the environment model and the perception is often confined to the cultural cognitive environment, and this is precisely beyond the human perception of the operational environment. However, the pattern of human reality is approximated and incomplete. Humans can understand the external world through cultural concept, which establishes the basis of methodology for neurophenomenology. It regards the assumed materialism as metaphysical, and regards the nature of knowledge of the world as epistemological idealism. Because of the cognitive environment, human beings can understand the physical external world or operational environment, which in turn reflects the principle of operational environment. In other words, in the cultural system, through the adaptation of culture to form a special human biological development process, it results in a special human cognitive model. 
Secondly, brain-based cognitive processes and behaviors are related to neuro-phenomenological epistemology and cognitive theory. Neuro-phenomenological methods are in a context of an evolutionary and bio-constructivist realism in which the organism regulates the reality (operational environment) by means of isomorphic structures and patterns. Materialistic meta-physics recognizes the correspondence between cognitive structures (the subjective structure of world knowledge) and the objective structure of the world, because cognitive structures are the result of biological evolution. The human experience and category should be adapted to the external environment, laying the foundation for some kind of correspondence between "thing-in-itself" and apparent forms [18]. Hypothetical realism is seen as an assumption of the external world independent of the spiritual world, but to a certain extent, human beings only understand the world in a special way. Since the formation of the cognitive world needs to be done with the processing of the organism, and the cognitive world must have the same principle as a functioning world. This cognitive environment stems from the lowest level of perceived abstraction. It is a symbolic relationship between object and the neural process, which is regulated by the cellular network. Mankind constructs a model of operational environment with meanings but these meanings are not sufficient and measurable in terms of special functions and cultural standards. Human cognition derives from our cultural system, and this is not just the spiritual image drawn from the material world. The causal control of the human brain transcends the physical boundaries.

Thirdly, the phenomenological approach to conscious research requires that the tradition of meditation be directly integrated into scientific rationality, but that there is a need to focus on the cognitive environment and the boundaries of the operational environment in the process of integration. Scientists recognize the special state of consciousness but reject introspection in a traditional sense, as this leads to a natural attitude that is subject to the confusion of hypothesis and prejudices [19]. In fact, through the introspection, this natural attitude can be deconstructed. Scientists trained by meditation are to verify their mental state by concentrating their attention. Meditation develops and maintains an independent objectivity field and the relative objectivity of its own phenomenon processing. Through meditation, they can directly understand the sensory hierarchy of consciousness, producing introspection of the nature of consciousness. In general, the methods of observation and experimentation require a particular state of the process, and the scientific concept of a particular state may require that scientists be able to enter all relevant stages of consciousness, thereby elevating the scientific subconscious observation procedure. In the Asian philosophy and religious system, meditation traditions are closely related to Buddhism, Hinduism and other religions, which is conducive to in-depth understanding of consciousness and to comply with the understanding and confirmation of the process of science. Mainstream epistemology in the future development of traditional consciousness research and modern scientific research may be derived from it, so as to give further insight into the reality of the general process of construction and self-consciousness of the world.

\section{REFERENCES}

[1] H.L. Dreyfus, "Intelligence Without Representation-Merleau-Ponty's critique of mental representation the relevance of phenomenology to scientific explanation." Phenomenology and the Cognitive Sciences 1.4 (2002), pp.367-383.

[2] S. Gallagher, and D. Zahavi, The phenomenological mind. London, pp. Routledge, 2013.

[3] E.Thompson, 'Mind in Life, pp. Biology, Phenomenology, and the Sciences of Mind. Belknap, Harvard. 2007

[4] B.Gupta, the Disinterested Witness. Evanston, IL, pp. Northwestern University Press, 1998.

[5] D.Dennett, "Are we explaining consciousness yet?" Cognition 79.1 (2001), pp.221-237.

[6] D.M. Rosenthal, "A theory of consciousness". In The Nature of Consciousness, N. Block et al.,Eds. Cambridge, MA, pp. MIT Press, 1997.

[7] J. Parvizi, and A. Damasio, "Consciousness and the brainstem". Cognition, 2001, 79:135-159.

[8] S.Gallagher, "Philosophical concepts of the self, pp. implications for cognitive science". Trends in Cognitive Sciences, 2000, 4:14-21.

[9] M. Winkelman, "Multidisciplinary Perspectives on Consciousness". Anthropology of Consciousness, 1994, 5(2), pp.16-25.

[10] I. Baruss, "Contemporary Issues Concerning the Scientific Study of Consciousness". Anthropology of Consciousness, 1992, 3(3-4), pp.28-35.

[11] S.Haack, Evidence and Inquiry (Evidence and inquiry ed.). Oxford, UK, pp. Blackwell, 1993.

[12] B.Aune, "Haack's Evidence and Inquiry". Philosophy and Phenomenological Research, 1996, 56 (3), pp.627-632.

[13] Laughlin, C. \& E. D’ Aquili, Biogenetic Structuralism. New York, pp. Columbia University Press, 1974.

[14] E. Thompson \& F. J. Varela, "Radical Embodiment: Neural Dynamics and Consciousness". Trends in Cognitive Sciences, 2001, 5 (10), pp.418.

[15] J. Piage, Biology and Knowledge. Chicago, IL, pp. University of Chicago Press, 1971.

[16] C.Laughlin, J. McManus \& E. d'Aquil, Brain, Symbol and Experience Toward a Neurophenomenology of Consciousness. New York, pp. Oxford University Press, 1992.

[17] C. Laughlin, Scientific Explanation and the Life World, pp. A Biogenetic Structural Theory of Meaning and Causation. Sausalito, CA, pp. Institute of Noetic Sciences, 1992.

[18] A. Danailov, \& C. Tiige, Theories of the Evolution of Knowing. Hillsdale, NJ, pp. Erlbaum, 1990.

[19] C. Laughlin, "Consciousness in Biogenetic Structural Theory". Anthropology of Consciousness, 1992a, 3(1, 2), pp.17-22. 